\title{
A CONCEPT OF MULTI-MODE HIGH SPECTRAL RESOLUTION LIDAR USING MACH-ZEHNDER INTERFEROMETER
}

\author{
Yoshitaka Jin $^{1 *}$, Nobuo Sugimoto ${ }^{1}$, Tomoaki Nishizawa ${ }^{1}$, Pablo Ristori ${ }^{2}$, and Lidia Otero ${ }^{2}$ \\ ${ }^{1}$ National Institute for Environmental Studies, 16-2 Onogawa, Tsukuba 305-8506 Japan, *Email: \\ jin.yoshitaka@nies.go.jp \\ ${ }^{2}$ División Lidar, CEILAP, UNIDEF (MINDEF - CONICET), UMI-IFAECI-CNRS 3351, Juan Bautista de \\ La Salle 4397 - B1603ALO Villa Martelli, Buenos Aires, Argentina
}

\begin{abstract}
In this paper, we present the design of a High Spectral Resolution Lidar (HSRL) using a laser that oscillates in a multi-longitudinal mode. Rayleigh and Mie scattering components are separated using a Mach-Zehnder Interferometer (MZI) with the same free spectral range (FSR) as the transmitted laser. The transmitted laser light is measured as a reference signal with the same MZI. By scanning the MZI periodically with a scanning range equal to the mode spacing, we can identify the maximum Mie and the maximum Rayleigh signals using the reference signal. The cross talk due to the spectral width of each laser mode can also be estimated.
\end{abstract}

\section{INTRODUCTION}

Observation of aerosol characterization is essential to understand the atmospheric radiation budget that affects on the climate change. High Spectral Resolution Lidar (HSRL) is an effective tool for quantitatively measuring aerosol extinction and backscattering coefficients with high sensitivity [1]. The extinction-tobackscattering ratio (lidar ratio) depends on the aerosol species, and therefore it is an important parameter for evaluating aerosol characteristics $[2$, 3].

HSRLs have been developed using Fabry-Perot interferometer [4], atomic filters [5], and Iodine filters [6]. Recently a method using tilted fieldwidened Michelson interference has been developed for an airborne HSRL [7]. HSRL can make daytime observations since the HSRL signal sensitivity is much higher than that of Raman lidar. HSRL is a key instrument for the next generation of aerosol lidar. However, traditional systems use single-longitudinal mode lasers that are costly because they need injection seeding.
Moreover, traditional HSRL tends to be complicated systems since it needs to control the receiver system and tune the laser wavelength. This study presents a measurement system and some simulation results for HSRL using lasers that oscillate in a multi-longitudinal mode (hereafter referred to as MM-HSRL). The basic concept of MM-HSRL is presented by Ristori et al. [8], and a lidar using a multimode laser and MZI has been developed by Bruneau et al. [9] for wind measurement. Our MM-HSRL system is both low cost and quite simple to operate compared to traditional systems.

\section{DESIGN OF A MM-HSRL SYSTEM}

A MM-HSRL measurement technique is briefly described bellow. Rayleigh scattering from atmospheric molecules has a broad spectrum due to the Doppler effect. In contrast, the Mie scattering spectrum is almost the same as the transmitted laser mode. Rayleigh scattering signals are separately measured by removing Mie scattering spectrum ranges with an interferometer. For MM-HSRL, the separate measurement can be achieved by using the realistic assumption that all mode spacings of the laser resonator are the same. The interferometer should have the same mode spacing as Mie scattering (i.e., identical to the free spectral range (FSR) of the transmitted laser).

To perform the measurement described above, we design the MM-HSRL system depicted in Fig. 1. The transmitter employs a multi-mode $\mathrm{Nd}$ :YAG laser at a second $(532 \mathrm{~nm})$ or third $(355 \mathrm{~nm})$ harmonic wavelength. A part of the transmitted laser light is injected into the telescope before being emitted into the atmosphere. The reason, as described later, is that the injected laser light is measured as a reference signal for evaluating the laser spectral properties. A high-speed $(>100 \mathrm{MHz}) \mathrm{A} / \mathrm{D}$ converter is 
employed to get the reference signals at the lowest height $(\mathrm{z}=0)$.

The receiver system employs a Mach-Zehnder interferometer (MZI) composed of a roof mirror mounted on a piezo stage and two half mirrors. The received light is divided into two beams with a beam splitter. The reflected light moves on to the movable roof mirror and back in the opposite direction, and again intersects with the penetrated light at the second beam splitter (i.e., interference occurs). The superimposed light rays after the MZI are measured with two photomultiplier tubes (PMT) for opposite phases. The optical path difference (OPD) of MZI is set at the position where the MZI FSR is identical to the laser-mode spacing. Controlling the piezo actuator of the roof mirror mount, the OPD of MZI changes on the order of nanometers. The OPD changes result in changes in wavelengths of constructive and destructive interference. We scan the MZI periodically with a scanning range equal to the mode spacing within several seconds. The displacement of OPD corresponds to the laser wavelength for the mode spacing.

By scanning the MZI, we can determine the optimal OPD for separating Mie and Rayleigh scattering components. The optimal OPD is determined by envelope analysis of the reference (transmitted laser) signal during the scanning. When we regulate the reference signal to minimum, each laser-mode wavelength is blocked at the OPD. Rayleigh (Mie) signals are obtained when destructive (constructive) interference occurs at each laser-mode wavelength. Mie scattering components are removed at the optimal $\mathrm{OPD}$, and the remaining Rayleigh components are measured for atmospheric signals. By setting the optimal OPD, Rayleigh and Mie scattering signals are simultaneously measured with each PMT because each MZI output is in the opposite phase.

The advantages of scanning are both to determine optimal OPD and to monitor bias caused by the laser spectral properties. Using the reference signal, we can estimate the cross talk due to the spectral width of each laser mode. The estimated cross talk is removed from the atmospheric signals. We can also monitor the displacement from optimal OPD for the reference signal and hence estimate the MZI efficiency depending on the displacement. This system does not need to know the absolute wavelength of laser modes. The important point is that the scan covers one or more mode intervals.

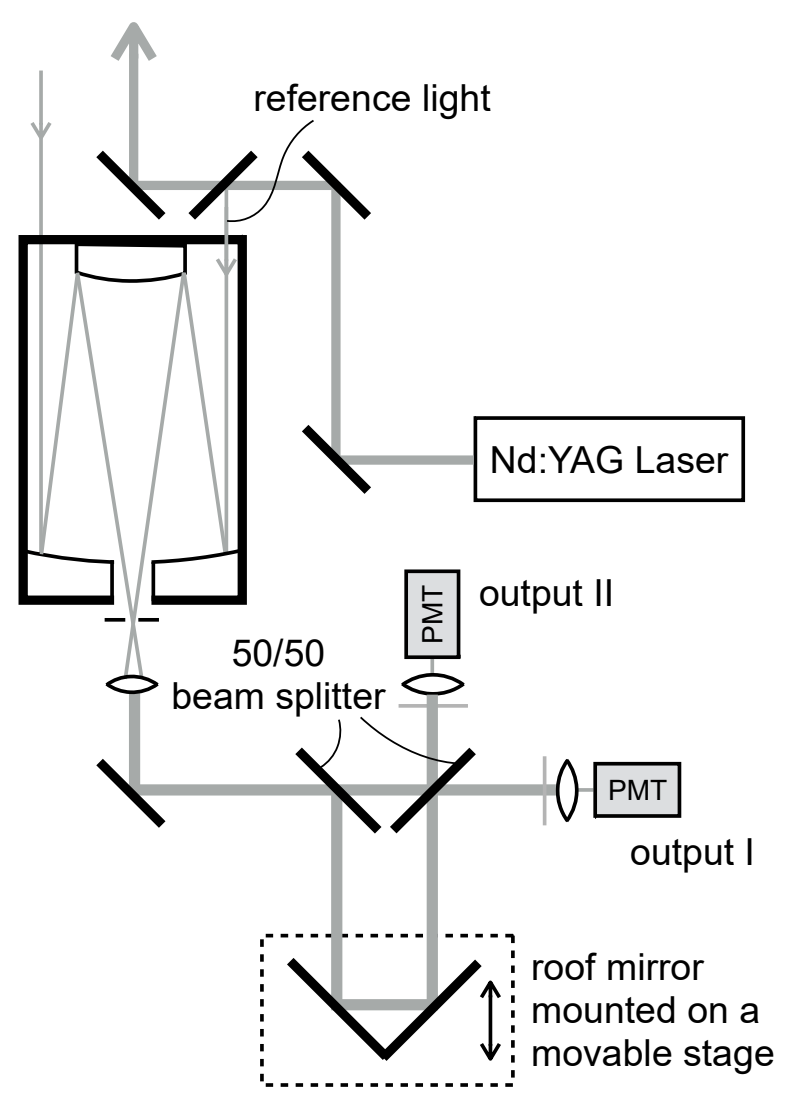

Fig. 1 System design of a MM-HSRL with a Mach-Zehnder Interferometer.

\section{PRELIMINARY SIMULATION RESULTS}

We will now present some simulation results for the MM-HSRL system. MZI output irradiance $I$ is expressed by as follows:

$$
I_{i}=2 r t \int_{-\infty}^{+\infty} I_{0}(k)\left[1+\cos \left(k d+\delta_{i}\right)\right] d k,
$$

where subscript $i$ denotes output 1 (parallel to the incident beam) or output 2 (perpendicular to the incident beam), $I_{0}(k)$ is the input irradiance, $\sqrt{r}$ and $\sqrt{t}$ are the reflectance and transmittance of the beam splitter, $k$ is the wave number, $d$ is the OPD, 
and $\delta$ is the phase shift by the beam splitters (here $\delta_{1}=0$ and $\left.\delta_{2}=\pi\right)$. The spectral broadening of each mode is determined from the reflectivity of laser cavity. The mode spacing is identical to the laser FSR, which depends on resonator length $L$ (i.e., $F S R=c / 2 L$, where $c$ is the speed of light).

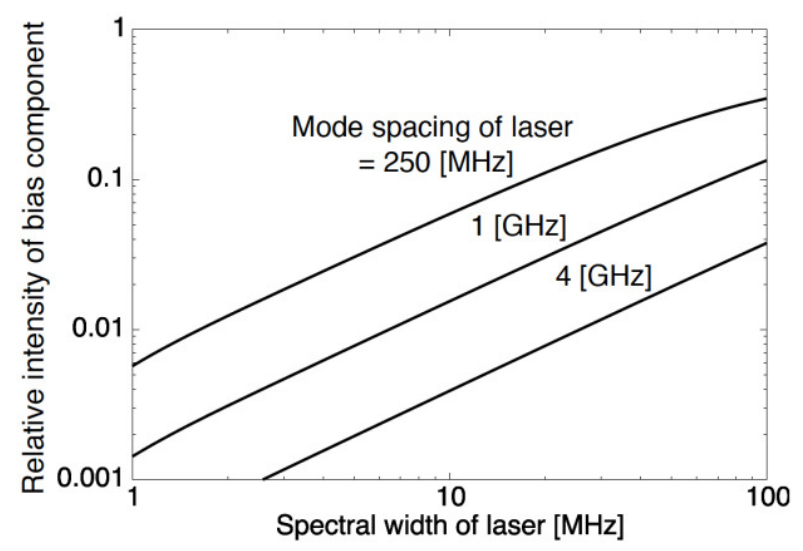

Fig. 2 Relative intensity of bias component for various spectral width and mode spacing of laser.

Using calculated output irradiance we can estimate the transmittance of aerosol and molecule signals as follows:

$$
T_{i, a}=\frac{I_{i, a}}{\int_{-\infty}^{+\infty} I_{0, a}(k) d k},
$$

and

$$
T_{i, m}=\frac{I_{i, m}}{\int_{-\infty}^{+\infty} I_{0, m}(k) d k}
$$

where subscripts $a$ and $m$ denote aerosols and molecules. We assume that aerosol signals have the same spectral properties as the laser. For molecular signals, the spectral width is set to $3 \mathrm{GHz}$ full-width half-maximum. The ratio $T_{a} / T_{m}$ is an index of efficiency of HSRL [7]. A low $T_{a} / T_{m}$ results in efficient removal of Mie signals from Mie+Rayleigh signals when detecting destructive interference.

The minimum $T_{a}$ (bias component) depends on the spectral width and mode spacing of laser (Fig.
2). Large spectral widths cause difficulties to remove Mie signals and increase the bias component. Therefore the efficiency of removing Mie signals is better when using the lasers with high reflectance mirrors. A short laser cavity (large FSR) results in a wide range of MZI spectrums for the removal of Mie-signals, resulting in decrease of bias component. Laser cavity length is related to an incident angle issue as below.

Some factors in the MM-HSRL system decrease $T_{a} / T_{m}$. Inclining the incident beam to the interferometer is a critical factor since this system applies large OPDs to match the MZI mode spacing to the laser FSR. We can calculate OPD at the incident angle $\theta$ as follows:

$$
O P D(\theta)=\frac{O P D\left(0^{\circ}\right)}{\cos (\theta)}
$$

$\triangle O P D\left(=O P D(\theta)-O P D\left(0^{\circ}\right)\right)$ increases with the incident angle (Fig. 3). The longer $O P D\left(0^{\circ}\right)$ causes a more rapid increase as expected. When $\triangle O P D$ is $0.5 \lambda$, the ratio $T_{a} / T_{m}$ is maximal since most aerosol signals are measured with constructive interference. Therefore the incident angle should be reduced to much less than the angle at $\triangle O P D=0.5 \lambda$.

The maximum incident angle to the interferometer is determined from the field stop of the telescope and the focal length of the collimator lens. If the beam divergence of the transmitted laser is $0.1 \mathrm{mrad}$ (after being expanded) and the focal length of the telescope is $2 \mathrm{~m}$, the field stop of the telescope should exceed $0.2 \mathrm{~mm}$. To constrain the maximum incident angle to $<0.5 \mathrm{mrad}$, the focal length of the collimator lens should exceed $200 \mathrm{~mm}$. Therefore, this system requires lasers with very low beam divergence. Inclining the incident beam causes other errors. Tilted rays cannot overlap perfectly at the second beam splitter of the MZI. The two rays (reflected and penetrated light in MZI) overlap geometrically about $95 \%$ at the intersection point if the incident angle is $1 \mathrm{mrad}$ and $O P D\left(0^{\circ}\right)$ is $40 \mathrm{~cm}$. For the area without overlap, extra signals are measured as leaking light without interference. 
This decreases the efficiency removing aerosol signals. Some other sensitivity studies are provided by Ristori et al. [8].
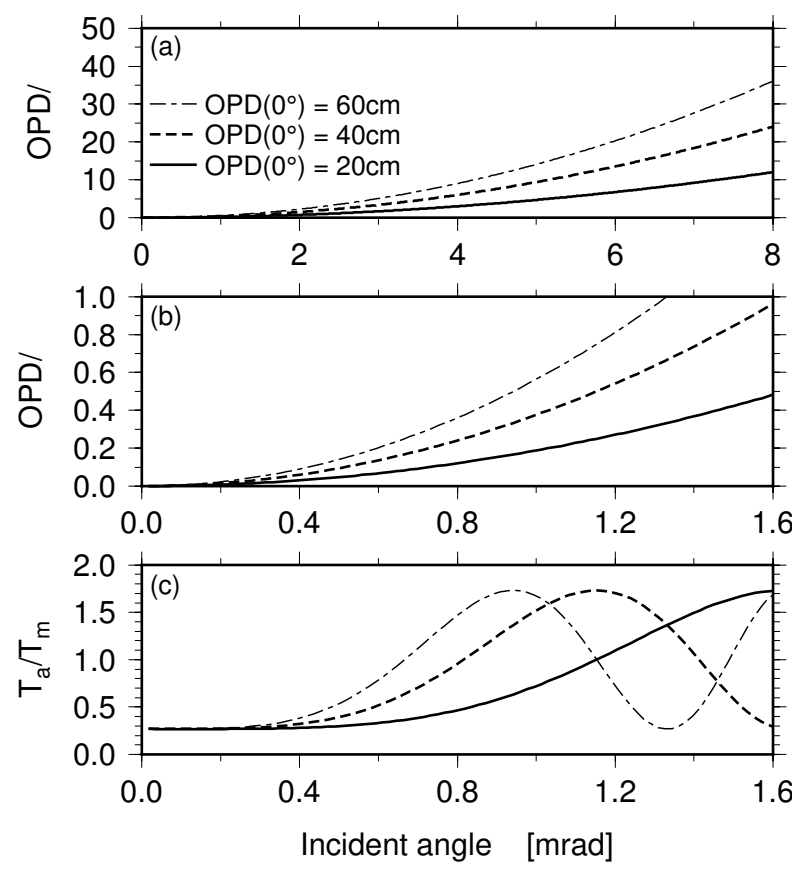

Fig. 3 Panels (a) and (b) plot OPD differences (unit laser wavelength) as a function of incident angle for three OPDs at $\theta=0^{\circ}$. Panel (b) is an expanded one of Panel (a). Panel (c) depicts the transmittance ratio as a function of the incident angle at laser finesse $=10$.

\section{FURTURE WORKS}

As future work, we will estimate system errors caused by inhomogeneous wave front at the field stop, spherical aberration of the collimator lens, and polarization dependence of beam splitters. We will also develop a MZI control method and a signal analysis algorithm. The ratio of reference signals from two channels will be used to adjust the roof mirror position. The reference signals can also be used as bias components to correct atmospheric signals.

\section{ACKNOWLEDGEMENT}

This research was supported by the Japan Science and Technology Agency (JST) / Japan International Cooperation Agency (JICA),
Science and Technology Research Partnership for Sustainable Development (SATREPS).

\section{REFERENCES}

[1] Nishizawa T., N. Sugimoto, and I. Matsui, 2010: Development of multi-wavelength highspectral resolution lidar system (2alpha+3beta+ 2delta), Proc. of 25th International Laser Radar Conference, 903-906.

[2] Liu Z., N. Sugimoto, and T. Murayama, 2002: Extinction-to-backscatter ratio of Asian dust observed by high-spectral-resolution lidar and Raman lidar, Applied Optics., 41(15), 2760-2767.

[3] Burton et al., 2012: Aerosol classification using airborne High Spectral Resolution Lidar measurements - methodology and example, Atmos. Meas. Tech., 5, 73-98.

[4] Fiocco G.G., G. Beneditti-Michelangeli, K. Maischberger, and E. Madonna, 1971: Measurement of Temperature and Aerosol to Molecule Ratio in the Troposphere by Optical Radar, Nature, 229, 78.

[5] Shimizu H., S. A. Lee, and C. Y. She, 1983: High spectral resolution lidar system with atomic blocking filters for measuring atmospheric parameters, Appl. Opt., 22, 1373-1381.

[6] Liu Z., Ichiro Matsui, and Nobuo Sugimoto, 1999: High-Spectral-Resolution Lidar Using an Iodine Absorption Filter for Atmospheric Measurements, Optical Engineering, 38(10) 16611670.

[7] Liu D., C. Hostetler, I. Miller, A. Cook, and J. Hair, 2012: System analysis of a tilted fieldwidened Michelson interferometer for high spectral resolution lidar, Opt. Express, 20, 14061420.

[8] Ristori et al., 2015: Development of a high spectral resolution lidar using a multi-mode laser and a tunable interferometer, Proc. of 27th International Laser Radar Conference.

[9] Bruneau et al., 2013: Direct-detection wind lidar operating with a multimode laser, Appl. Opt., 52, 4941. 